\title{
Trajectory Tracking of a Nonholonomic Wheeleed Mobile Robot Using Hybrid Controller
}

\author{
Zain Anwar Ali, Daobo Wang, Muhammad Safwan, Wanyue Jiang, and Muhammad Shafiq
}

\begin{abstract}
This paper address a novel method for nonholonomic Wheeled Mobile Robot (NWMR) trajectory tracking using a hybrid controller. In the essential of the proposed scheme, hybrid algorithm includes fuzzy logic based Regulation, Pole-Placement and Tracking (F-RST). The hybrid controller easily track the reference trajectory and linearize the nonlinear behavior of robot under the influence of uncertainty and disturbance. To ensure the efficiency of suggested controller the performance of hybrid controller is compared with the classical RST and Back-stepping controller. Simulation results shows that the control strategy is effectively work over nonlinear simulations of kinematic and dynamic model of WMR and system response shows a good convergence and robustness towards stability with zero steady-state error and very less transient error.
\end{abstract}

Index Terms-Wheeled mobile robot, RST controller, fuzzy base RST controller.

\section{INTRODUCTION}

Previously, for mobile robots motion control, direction and trajectory tracking is considered a challenging task [1]. And found a great interest of the researchers all over the world. Mobile robots are widely use in the fields of medicine, health care and manufacturing [2]. A WMR is a nonlinear multiple inputs and multi outputs (MIMO) system [3]. With respect to their mechanical design, robots are categorize into holonomic and nonholonomic robots. In Holonomic robots, controllable degree of freedom is same as controllable inputs, while nonholonomic WMR turns are more valuable due to fact that they offer less input control [4].

Trajectory tracking is the basic goal of mobile robot because a robot takes a decision where to go and that information is taken by a reference path or leader robot. The problem of tracking can be divided into dynamic and kinematic tracking control model. Kinematics controller objective is to produce velocity as output to converge the desired and reference trajectories to zero. When the velocity of system varies or noises creep in, the controller's performance decreases. Modeling errors produce technical hitches in effective control of mobile robots. These situations demands dynamic model of mobile robots.

Several control algorithms are presented by different researchers for the trajectory tracking of WMR. In [5] the

Manuscript received April 10, 2016; revised June 12, 2016.

Zain Anwar Ali, Daobo Wang, and Wanyue Jiang are with College of Automation Engineering, Nanjing University of Aeronautics and Astronautics, Nanjing, China (e-mail: zainanwar86@hotmail.com).

Muhammad Safwan and Muhammad Shafiq are with the Electronic Engineering Department of Sir Syed University of Engineering and Technology, Karachi, Pakistan. kinematic equations behaves as a control trouble and in [6] only the dynamic model along with velocity saturation and interruption adaptability limitations are measured simultaneously to track the trajectory of a nonholonomic WMR. A doubled close loop control scheme is presented to achieve a stability. The inner loop is based on integral Sliding Mode Controller (SMC) while the outer loop have a tangent function of hyperbola. In reference [3] Dual Proportional Integral Derivative (D-PID) controllers were designed for controlling the two wheels individually whereas, twin input and single output was controlled by nine rules.

In [7] and [8] trajectory tracking of nonholonomic robotic system have been presented in which PID based adaptive tracking controller was used to track the trajectory, velocity tracking, stability of position and control law was proved by Lyapunov stability theorem. Previously in reference [9] the back-stepping based controller with Lyapunov stability is used to track the spherical BHQ-2 robot dynamic model and it's depend on the equations of Boltzmann-Hamel.

Previously many control methods were previously used but in this research we are using a new mathematical approach of fuzzy based RST controller. The RST control algorithm is a classical approach which is based on poleplacement theory [10] by analyzing its characteristics in our robot classical RST controller applied directly on the system and secondly Fuzzy based RST strategy is applied in our model by taking the difference of their robustness between both controllers [11], [12]. However the RST controller appears to be remarkable substitute to Proportional Integral (PI) controller, because it permits finest conciliation between speed and accuracy.

The key contributions of the research are: (1) A stable controller with tuning gains of RST; (2) Basic controller with advance adaptive conditions with respect to time and tune it-self's (3) Settling time of our proposed controller is quite better as compared to previous back-stepping algorithm of [19] and classical RST method; (4) The stability and robustness of controller is proven in the results.

In this research we are taking the dynamic as well as kinematic model of WMR. By extending the approach in [13] a Fuzzy based RST controller is employed to control the kinematic model of mobile robot, as compared to the previous research, we are using new control algorithm to control the kinematic as well as dynamic model of a WMR. The accuracy and precision of our work is to be compare with the classical RST and Back-stepping controller that shows the efficiency of our suggested method.

The rest of paper is distributed in to five sections. In which Section II defines the brief dynamic and the kinematic model of a WMR. The control algorithm of 
nonholonomic mobile robot is present in Section III and Section IV have simulation and discussion results and in last section concluded the whole paper.

\section{Modeling of WheEled MobILE Robot}

The robot model is divided in to two sub models i-e kinematic and dynamic model. The model of nonholonomic WMR has three degree of freedom (3-DOF) in the presence of disturbance and uncertainties which is shown by the position in figure 1 [15]. The mechanical model of WMR is shown in which two wheels is attached in an axle and in the front of WMR a passive wheel is fixed. The distance between both wheels in denoted by " $2 \mathrm{R}$ " (radius of a wheel), while the distance between point " $\mathrm{P}$ " and " $\mathrm{C}$ " (the center of mass) is " $d$ "

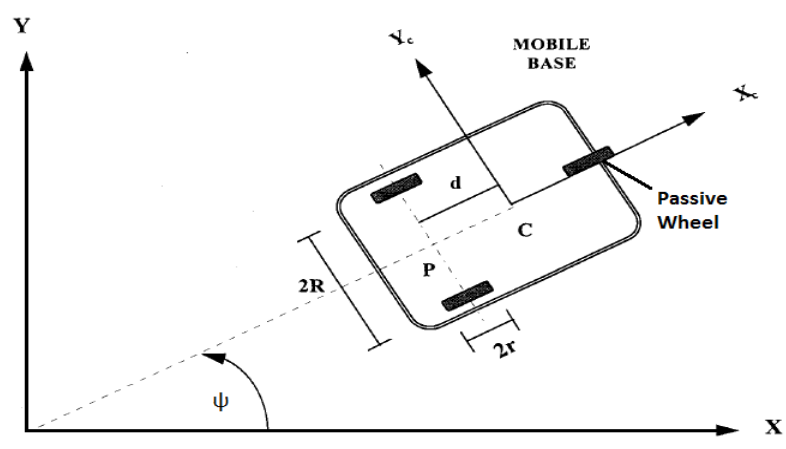

Fig. 1. Model of nonholonomic wheeled mobile robot.

\section{A. Kinematic Model}

The reference coordinate are $\{O, X, Y\}$ and the position of robot is defined by vector $\xi(t) \in \mathfrak{R}^{m \times 1}$ in equation 1 .

$$
\xi(t)=[x(t) y(t) \Psi(t)]^{T}
$$

where $\Psi(t)$ is leading direction taken counterclockwise and $[x(t), y(t)]$ the center of mass of the robot $(\mathrm{X}, \mathrm{Y})$ coordinate with respect to time.

$$
\dot{\xi}(t)=\left[\begin{array}{c}
\dot{x} \\
\dot{y} \\
\dot{\Psi}
\end{array}\right]=\left[\begin{array}{cc}
\cos \Psi(t) & 0 \\
\sin \Psi(t) & 0 \\
0 & 1
\end{array}\right]\left[\begin{array}{c}
v_{l}(t) \\
w_{a}(t)
\end{array}\right]+\left[\begin{array}{c}
d_{n}(t) \\
d_{u n}(t)
\end{array}\right] t \geq 0
$$

In which the linear velocity is denoted by $v_{l}(t), w_{a}(t)$ is the angular velocity. Disturbances formed by arbitrary noise and model uncertainties are denoted by $d_{n}(t), d_{u n}(t)$.

$$
\dot{\xi}(t)=J(\xi) v_{j}(t)+d_{t}(t) \quad t \geq 0
$$

The Jacobean matrix $J(\xi)$ transform the input velocities to robotic velocities and $d_{t}(t)$ sum-up both noises as.

$$
d_{t}(t)=\left[d_{n}(t), d_{u n}(t)\right]
$$

The reference trajectory with respect to time is written by the kinematic controller model.

$$
\xi_{r t}(t)=\left[x_{r t}(t) y_{r t}(t) \Psi_{r t}(t)\right]^{T}
$$

To design our kinematic controller such that a path where position error and orientation of robot error goes to zero with respect to time, hence trajectory error is described by the equation.

$$
\begin{gathered}
\xi_{t e}(t)=\left[\begin{array}{l}
e_{1}(t) \\
e_{2}(t) \\
e_{3}(t)
\end{array}\right]=\left[\begin{array}{c}
t e_{x}(t) \\
t e_{y}(t) \\
t e_{\Psi}(t)
\end{array}\right] \\
=\left[\begin{array}{ccc}
\cos \Psi(t) & \sin \Psi(t) & 0 \\
-\sin \Psi(t) & \cos \Psi(t) & 0 \\
0 & 0 & 1
\end{array}\right]\left[\begin{array}{l}
x_{r t}(t)-x(t) \\
y_{r t}(t)-y(t) \\
\Psi_{r t}(t)-\Psi(t)
\end{array}\right]
\end{gathered}
$$

The derivative of equation (6) gives.

$$
\begin{gathered}
\xi_{t e}(t)=\left[\begin{array}{c}
t \dot{e}_{x}(t) \\
t \dot{e}_{y}(t) \\
t \dot{e}_{\Psi}(t)
\end{array}\right] \\
{\left[\begin{array}{c}
-v_{l}(t)+w_{a}(t) t e_{y}(t)+v_{r t}(t) \cos t e_{\Psi}(t) \\
-w_{a}(t) t e_{x}(t)+v_{r t}(t) \sin t e_{\Psi}(t) \\
-w_{a}(t)+w_{r t}(t)
\end{array}\right]}
\end{gathered}
$$

From reference [20] the angular and linear velocities are defined as below.

$$
\begin{array}{r}
w_{r t}(t)=\dot{x}_{r t}(t) \ddot{y}_{r t}(t)-\dot{y}_{r t}(t) \ddot{x}_{r t}(t) / \dot{x}_{r t}^{2}(t)+\dot{y}_{r t}^{2}(t) \\
v_{r t}(t)=\left(\dot{x}_{r t}^{2}(t)+\dot{y}_{r t}^{2}(t)\right)^{1 / 2}
\end{array}
$$

\section{B. Dynamic Model}

The nth dimensional space configuration (q) dynamics of robot is taken from [16-18]. In which famous Lagrange formulation is utilized to describe the dynamic model.

$$
\begin{array}{r}
M(q) \ddot{q}+v_{t}(q, \dot{q}) \dot{q}+F(\dot{q})+G(q)+\tau_{d}+A^{T}(q) \lambda= \\
B(q) \tau
\end{array}
$$

where $M(q) \in \mathfrak{R}^{n \times n}$ is a inertial matrix which is symmetric positive definite, $v_{t}(q, \dot{q}) \in \mathfrak{R}^{n \times n}$ is the centripetal and coriolis matrix, the surface friction is defined by $F(\dot{q}) \in$ $\mathfrak{R}^{n \times n}$, the gravitational vector $G(q) \in \mathfrak{R}^{n \times 1}$, Unknown disturbance is denoted by $\tau_{d}$, the input $\mathrm{B}(\mathrm{q})$ is the transformation matrix $B(q) \in \mathfrak{R}^{n \times r}, \quad A(q) \in \mathfrak{R}^{n \times m}$ nonholonomic constraints and Lagrange multiplier constraints is defined by $\lambda \in \mathfrak{R}^{1 \times m}$. The variables in (10) is expressed in matrix form as,

$$
\begin{gathered}
M(q)=\left[\begin{array}{ccc}
m & 0 & 0 \\
0 & m & 0 \\
0 & 0 & I
\end{array}\right], v_{t}(q, \dot{q})=0, G(q)=0, \\
\tau=\left[\begin{array}{l}
\tau_{r} \\
\tau_{l}
\end{array}\right], \\
A^{T}(q)=\left[\begin{array}{c}
-\sin \Psi(t) \\
\cos \Psi(t) \\
-d
\end{array}\right] \\
B(q)=(1 / r)\left[\begin{array}{cc}
\cos \Psi(t) & \cos \Psi(t) \\
\sin \Psi(t) & \sin \Psi(t) \\
R & -R
\end{array}\right] \\
\lambda=-m(\dot{x} \cos \Psi(t)+\dot{y} \sin \Psi(t)) \dot{\Psi}
\end{gathered}
$$

Earlier in [14], it is shown that robot can maneuver in the course of castor wheel, i.e, the robot verifies the stipulation of no slipping and pure rolling. The constraint of WMR is 
expressed as.

$$
\begin{aligned}
& \dot{y}(t) \cos \Psi(t)=d \dot{\Psi}+\dot{x}(t) \sin \Psi(t) \\
& \text { Therefore } A(q)=\left[\begin{array}{lll}
-\sin \Psi(t) \cos \Psi(t) & 0
\end{array}\right]^{T}
\end{aligned}
$$

From equation (12) all the kinematic restrictions are taken to be sovereign of time and written as.

$$
A(q) \dot{q}=0
$$

To consider $S(q)$ a matrix having full rank formed by independent vector having linear fields and null space of $A(q)$ is written by

$$
A(q) S(q)=0
$$

According to equation (13) and (14) the Vector time function $v_{l}(t) \in \mathfrak{R}^{n} \forall t$.

$$
\dot{q}=S(q) v_{l}(t)
$$
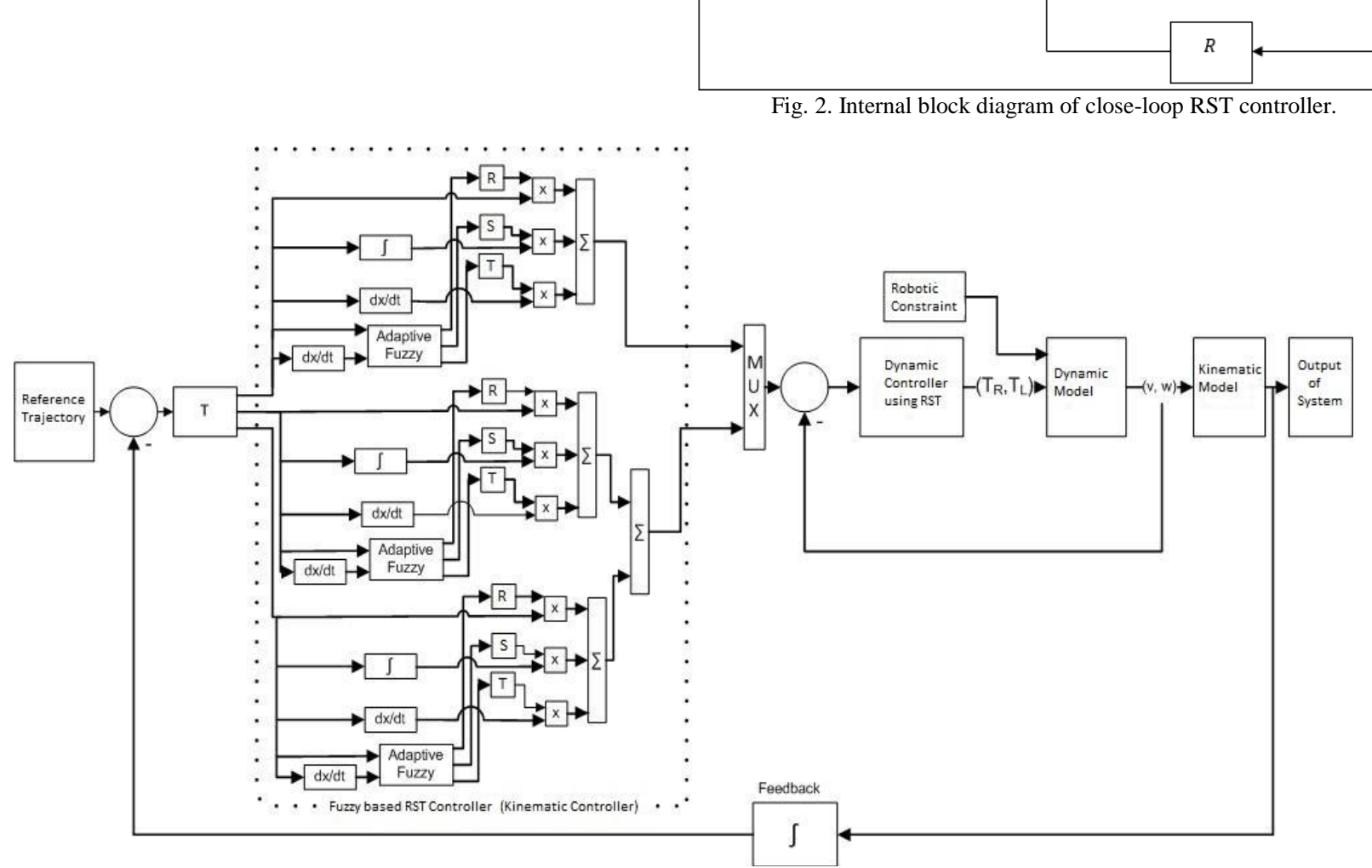

Fig. 3. Trajectory tracking block diagram of fuzzy based RST controller.

\section{A. Feed-Forward Algorithm}

The error and reference trajectory are used to make the feed-forward velocities. From reference [17] the particular equation describes the controlling of feed-forward motion of WMR is.

$$
v_{f f d}(t)=\left[\begin{array}{c}
v_{f f d}(t) \\
w_{f f d}(t)
\end{array}\right]=\left[\begin{array}{c}
v_{r t}(t) \cos t e_{\Psi}(t) \\
w_{r f}(t)
\end{array}\right]
$$

Therefore $v_{r t}(t)$ and $w_{r f}(t)$ are reference linear and angular velocities respectively. Whereas the feed-forward motion controller treated in terms of velocity error which was produced by the reference signal and earlier control signal.

\section{CONTROL Algorithm}

Our proposed controller algorithm is further divided in to two feed-forward and feed-backward control algorithms, which are described below. The generalize equation of our proposed algorithm is.

$$
v_{c}(t)=\left[\begin{array}{c}
v_{l}(t) \\
w_{a}(t)
\end{array}\right]=\left[\begin{array}{c}
v_{f f d}(t) \\
w_{f f d}(t)
\end{array}\right]+\left[\begin{array}{c}
v_{f b w}(t) \\
w_{f b w}(t)
\end{array}\right]
$$

In which $v_{f f d}(t)$ and $w_{f b w}(t)$ are linear and angular feedforward velocities, similarly the linear and angular back-ward velocities are $v_{f b w}(t)$ and $w_{f b w}(t)$.

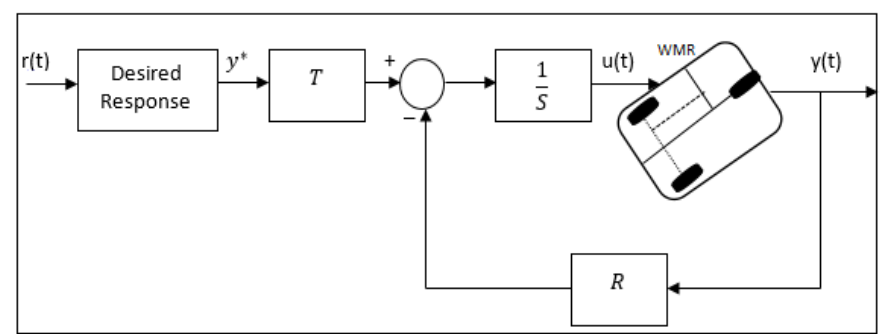

Fig. 2. Internal block diagram of close-loop RST controller.

$$
v_{e r}(t)=v_{f f d}-v_{f b w}
$$

\section{B. Feed-Backward Algorithm}

The kinematic feed-backward controller which is based on Fuzzy RST controller is defined in this section, in which the RST control mechanism control the kinematic controller and the gains of RST controller is used for the tuning of fuzzy logic. This dual controller based hierarchy is utilized for the fine tuning of each input. The close-loop RST control strategy and schematic of proposed controller are given in Fig. 2 and Fig. 3 respectively.

In Fig. 3 the overall system model and control algorithm is shown. The linguistics levels of the fuzzy based RST controller are assigned as (NB) negative below, (NS) negative Small, (Z) zero, (PS) positive small and (PB) 
positive big, in which " $\mathrm{e}$ " is the error and " $d e / d t$ " is its derivative having ranges between -1 to 1 . The fuzzy logic control if-then rules are defined in table 1-3 while fuzzy logic input membership functions are shown in Fig. 4 and Fig. 5 for KR, KS and KT.

TABLE I: FUZZY CONTROLLER LOGIC (IF-THEN) RULE FOR KR

\begin{tabular}{|c|c|c|c|c|c|}
\hline $\overrightarrow{d e / d t}$ & NB & NS & Z & PS & PB \\
\hline error $\downarrow$ & & & & & \\
\hline NB & PB & PC & PC & PS & Z \\
\hline NS & PC & PC & PS & Z & NS \\
\hline Z & PC & PS & Z & NS & NC \\
\hline PS & PS & Z & NS & NC & NB \\
\hline PB & Z & NS & NC & NB & NB \\
\hline
\end{tabular}

TABLE II: FUZZY CONTROLLER LOGIC (IF-THEN) RULE FOR KS

\begin{tabular}{|c|c|c|c|c|c|}
\hline $\overrightarrow{d e / d t}$ & NB & NS & Z & PS & PB \\
\hline error $\downarrow$ & & & & & \\
\hline NB & PB & PB & PS & PS & Z \\
\hline NS & PB & PS & PS & Z & NS \\
\hline Z & PS & PS & Z & NS & NS \\
\hline PS & PS & Z & NS & NS & NB \\
\hline PB & Z & NS & NS & NB & NB \\
\hline
\end{tabular}

TABLE III: FUZZY CONTROLLER LOGIC (IF-THEN) RULE FOR KT

\begin{tabular}{|c|c|c|c|c|c|}
\hline $\overrightarrow{d e / d t}$ & NB & NS & Z & PS & PB \\
\hline error $\downarrow$ & & & & & \\
\hline NB & PB & PB & PS & PS & Z \\
\hline NS & PB & PS & PS & Z & NS \\
\hline Z & PS & PS & Z & NS & NS \\
\hline PS & PS & Z & NS & NS & NB \\
\hline PB & Z & NS & NS & NB & NB \\
\hline
\end{tabular}

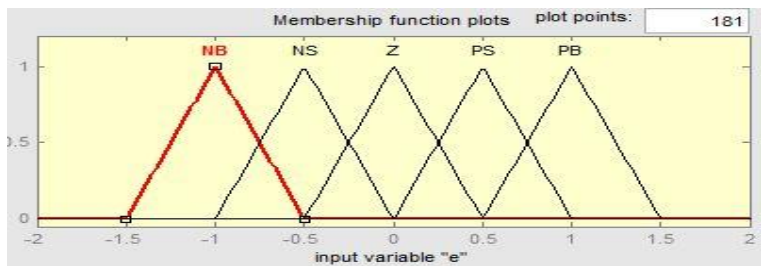

Fig. 4. Input error membership function of fuzzy logic.

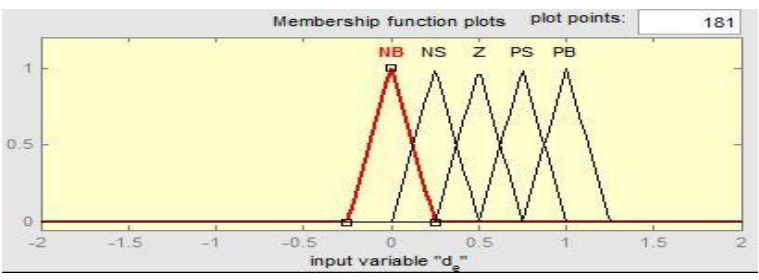

Fig. 5. Input derivative error membership function of Fuzzy logic.

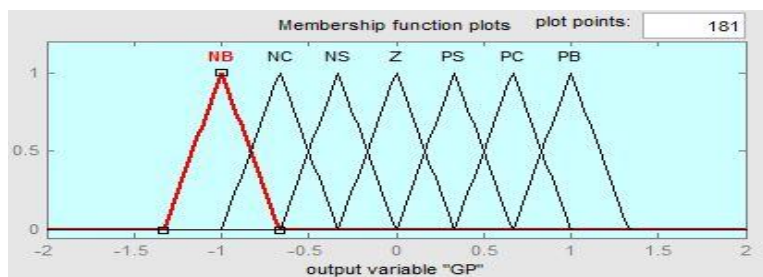

Fig. 6. Output regulation gain (FKR) memberships function of fuzzy logic.

The fuzzy logic controller outputs are Fuzzy Regulation gain (FKR), Fuzzy Pole-Placement gain (FKS) and Fuzzy Tracking gain (FKT). In which, linguistic level are assigned as zero (ZE), Positive Small (PS) and Positive Big (PB), and their defined ranges are among 0 to1. Hence output of fuzzy logic controller are transformed to a crisp output by defuzzification and shown in Fig. 6 to Fig. 8.

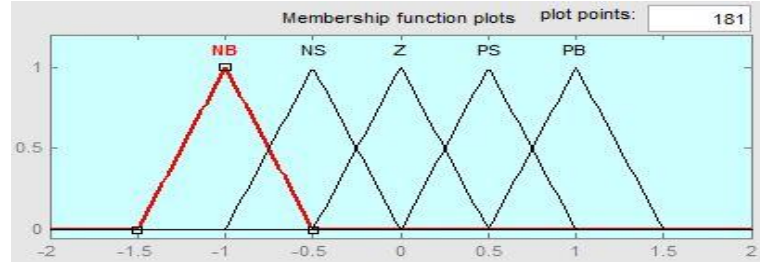

Fig. 7. Output pole-placement Gain (FKS) memberships function of fuzzy logic.

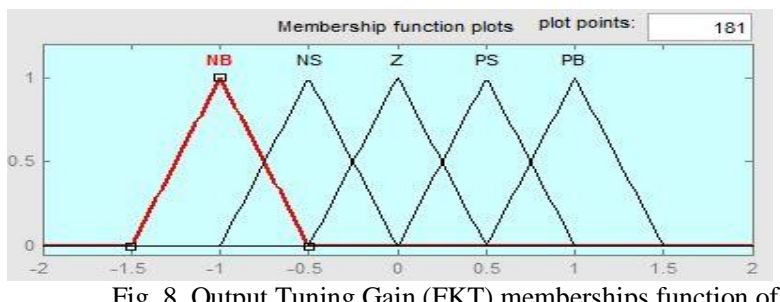

Fig. 8. Output Tuning Gain (FKT) memberships function of Fuzzy logic

Following are the results of fuzzy logic controllers used to tune adaptive gain for the RST controller and written as,

$$
\begin{array}{r}
u_{F R}(t)=f_{R}\left(e_{x}, \frac{d e_{x}}{d t}\right) G_{R} e_{x}(t) \\
u_{F S}(t)=f_{S}\left(e_{x}, \frac{d e_{x}}{d t}\right) G_{S} \int_{0}^{t} e_{x}(t) \\
u_{F T}(t)=f_{T}\left(e_{x}, \frac{d e_{x}}{d t}\right) G_{T} \frac{d e_{x}(t)}{d t}
\end{array}
$$

whereas the Regulation gain $\left(G_{R}\right)$, Pole-Placement gain $\left(G_{S}\right)$ and Tracking gain $\left(G_{T}\right)$ of our proposed RST controller is given in equation (19-21). Such that the values of gain are tuned manually, after that the precise tuning of the system is performed by $F K_{R}, F K_{S}$ and $F K_{T}$.

and,

$$
\begin{gathered}
F K_{R}=f_{R}\left(e_{x}, \frac{d e_{x}}{d t}\right) ; F K_{S}=f_{S}\left(e_{x}, \frac{d e_{x}}{d t}\right) ; \\
F K_{T}=f_{T}\left(e_{x}, \frac{d e_{x}}{d t}\right)
\end{gathered}
$$

Finally, the equation of Fuzzy based RST (F-RST) controller to control nonholonomic WMR is written as:

$$
U_{F-R S T_{X, Y, \Psi}}=F\left[\frac{T}{R}\right] *(U c(t))-F\left[\frac{S}{R}\right] Y(t)
$$

In which RST is defined as,

$$
\begin{gathered}
R=\left(q^{2}+r_{0} q+r_{1}\right) ; \\
S=\left(S_{0} q^{2}+S_{1} q+S_{2}\right) ; \\
T=\left(T_{0} q+T_{1}\right) A_{0}
\end{gathered}
$$

\section{Simulation AND Results}

In this section our proposed Fuzzy based RST controller is applied on both kinematic and dynamic model of robot. The justification and authentication of both models are simulated on Simulink Matlab by using "infinity" shaped 
reference input for the controllers. In referenced, input the linear and angular velocities are changed constantly, which help us to analyze the performance of our controller in the existence of model uncertainty and disturbance. While, in this paper the accuracy and precision of our controller is compared with simple RST controller and Back-stepping control method of [19].

For simulation, our robot parameters are $r=0.03 \mathrm{~m}, R=$ $0.15 \mathrm{~m}, I=2.5 \mathrm{kgm}^{2}$ and $m=4 \mathrm{~kg}$. Initially, robot is aligned at $\xi(t)=\left[\begin{array}{lll}0 & 0 & 0\end{array}\right]^{T}$ and the same inputs are applied to other three control algorithms, in which our anticipated controller displays enhanced tracking accuracy and indeed track the reference trajectory as compared to other controllers. The trajectory tracking performance is shown in Fig. 9 in which we neglect the angular and linear velocities. Fig. 10 shows the external noise of our system.

Fig. 11 and Fig. 12 shows the "X" and "Y" position error and Fig. 13 shows the " $\Psi$ " direction error of our robot respectively. In " $\mathrm{X}$ " location our proposed F-RST and Back-stepping controller converges to zero and stabilize at 0.3 seconds and simple RST controller stabilize in 0.9 seconds and for the "Y" location position error the F-RST and Back-stepping again stabilize at 0.3 seconds, a fear amount of time and simple RST stabilize at 1.5 seconds, so we can observe that F-RST and Back-stepping controller have faster stability and very less transient error as compared to the simple RST controller having very slow convergence towards stability and has large transient error. Lastly figure 13 shows the " $\Psi$ " direction or rotational error of our robot in which F-RST and RST controller linearize towards stability at 0.1 seconds, while the Back-stepping controller have overshoot and undershoot issues which is not good for the practical implementation point of view that is clearly shown in Fig. 13 and it will stabilize at 0.14 seconds it is also shown in Fig. 9 that Back-stepping controller not full fill the reference trajectory successfully due to the rotational error.



Fig. 9. Comparison Results of our proposed F-RST with RST and Backstepping controller.

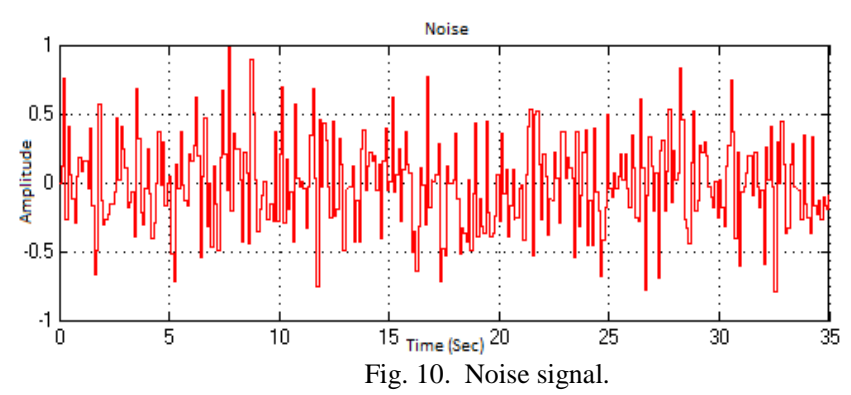

Simulation results demonstrate the efficiency of our controller along with simple RST and back-stepping controller. The error plots of our system is shown in figure 11-13, that shows the F-RST controller contains very low chattering, settling time and steady state error along $\mathrm{X}, \mathrm{Y}$ and $\Psi$ co-ordinates for the robot.

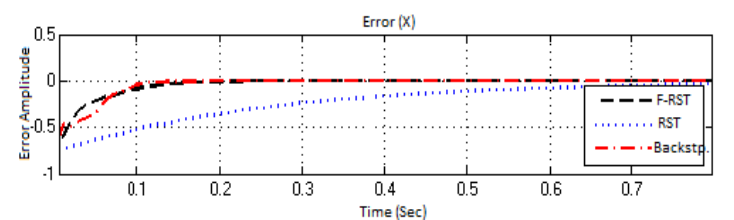

Fig. 11. Plotting of error "X" with our proposed F-RST with RST and back-stepping controller.

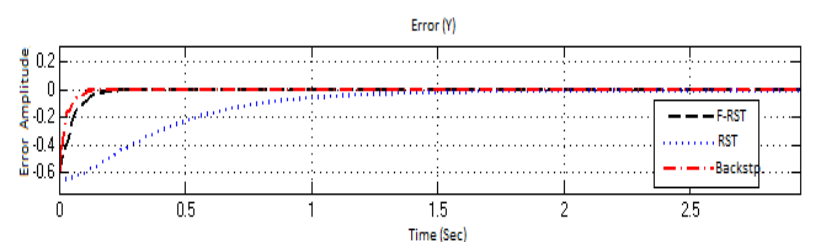

Fig. 12. Plotting of error "Y" with our proposed F-RST with RST and back-stepping controller.

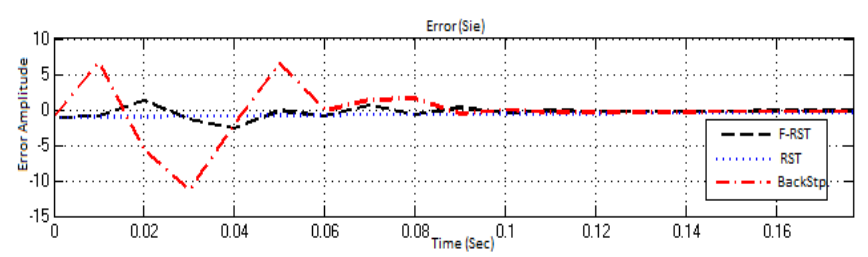

Fig. 13. Plotting of error " $\Psi$ " with our proposed F-RST with RST and back-stepping controller.

\section{CONCLUSION}

In this research, a new mathematical approach of Fuzzy based RST control strategy is proposed for controlling the nonlinear behavior of WMR model along with model uncertainties and system disturbance. A feed-forward and feed-backward control algorithm is presented separately for the trajectory tracking of WMR. To check the robustness of our controller we compare F-RST with RST and Backstepping controller. The precision and accuracy of our algorithm is proved by using Matlab Simulink model that shows better accuracy, speed and less transient error. Moreover we can say that our proposed algorithm have good convergence and robustness towards stability in less amount of time along with zero steady state error.

\section{ACKNOWLEDGEMENT}

This research work is supported by National Natural Science Foundation of China (NNSFC) and the grant number is 61503185 .

\section{REFERENCES}

[1] M. Asif, M. J. Khan and N. Cai, "Adaptive sliding mode dynamic controller with integrator in the loop for nonholonomic wheeled mobile robot trajectory tracking," International Journal of Control, 2014.

[2] N. B. Hoang and H. J. Kang, "Observer-based dynamic parameter Identification for wheeled mobile robots," International Journal of Precision Engineering and Manufacturing, vol. 16, no. 6, pp. 10851093.

[3] M. H. Khooban, A. Alfi, and D. N. M. Abadi, "Teaching-learningbased optimal interval type-2 fuzzy PID controller design: A 
nonholonomic wheeled mobile robots," Robotica, vol. 31, pp. 10591071, 2013.

[4] Y. N. Wang, Z. Q. Miao, H. Zhong, and Q. Pan, "Simultaneous stabilization and tracking of nonholonomic mobile robots: A lyapunov-based approach," Journal of Robotic Systems, vol. 14, no. 3, pp. 149-163, 1997.

[5] P. S. Tsai, L. S. Wang, and F. R. Chang, "Modeling and hierarchical tracking control of tri-wheeled mobile robots," IEEE Transactions on Robotics, vol. 22, no. 5, 2006.

[6] M. Yue, G. Q. Wu, S. Wang, and C. An, "Disturbance observer based trajectory tracking control for nonholonomic wheeled mobile robot subject to saturated velocity constraints," Applied Artificial Intelligence, vol. 28, pp. 751-765, 2014.

[7] H. Chang and T. Jin, "Adaptive tracking controller based on the PID for mobile robot path tracking," Springer-Verlag Berlin Heidelberg, 2013.

[8] K. Shojaei, A. M. Shahri, and B. Tabibian, "Design and implementation of an inverse dynamicscontroller for uncertain nonholonomic robotic systems," J Intell Robot Syst, 2013, vol. 71, pp. 65-83.

[9] Z. Qiang, Z. B. Liu, and C. Yao, "A back-stepping based trajectory tracking controller for a non-chained nonholonomic spherical robot," Chinese Journal of Aeronautics, vol. 21, pp. 472-480, 2008.

[10] A. Cuenca and J. Salt, "RST controller design for a non-uniform multi-rate control system, " Journal of Process Control, 2012.

[11] Z. A. Ali, D. B. Wang, and M. Aamir, "Design a robust RST controller for stabilization of a tri-copter UAV," Pakistan Journal of Engineering Technology and Science, vol. 5, no. 1, 2015.

[12] Z. A. Ali, D. B. Wang, R. Javed, and A. Aklbar, "Modeling and controlling the dynamics of tri-rotor UAV using robust RST controller with MRAC adaptive algorithm," International Journal of Control and Automation, vol. 9 , no. 3, 2016.

[13] M. Safwan, V. Uddin, and M. Asif, "Nonholonomic mobile robot trajectory tracking using hybrid controller," Mehran University Research Journal of Engineering and Technology.

[14] R. Fierro and F. L. Lewis, "Control of a nonholonomic mobile robot: Backstepping kinematics into dynamics," in Proc. the 34th IEEE Conference on Decision and Control, pp. 3805-3810.

[15] E. Freund and R. Mayr, "Nonlinear path control in automated vehicle guidence," IEEE Transaction on Robotics and Automation, vol. 13, pp. 49-60, 1997.

[16] T. K. Proychev, "Fuzzy-net control of nonholonomic mobile robot using evolutionery feedback error learning," Robotics and Autonomous Systems, 1998.

[17] D. N. Kar and Chaudhury, "Simple neuron based adaptive controller for a nonholonomic mobile robot including actuator dynamics," Neurocomputing, 2006.

[18] W. S. Lin, C. L. Huang, M. K. Chuang, "Hierarchical fuzzy control for autonomous navigation of wheeled robots," IEEE Proceedings: Control Theory and Applications, vol. 152, 2005, pp. 598-606.

[19] R. Fierro and F. L. Lewis, "Control of a nonholonomic mobile robot: Backstepping kinematics into dynamics," Journal of Robotic Systems, vol. 14, no. 3, pp. 149-163, 1997.

[20] R. Fierro and F. L. Lewis, "Control of a nonholonomic mobile robot using neural networks," IEEE Transactions on Neural Networks, vol. 9, pp. 589-600, 1998.



Technology.



Daobo Wang is a professor in the College of Automation Engineering at Nanjing University of Aeronautics and Astronautics, China. He was an official designated visiting scholar at University of Bath, UK from 1987 to 1989 and senior visiting scholar at University of Waterloo, Ontario, Canada in 1999. His research interests include unmanned aerial vehicle flight control and aeronautical simulation.

Muhammad Safwan received his B.S. degree in electronic engineering from the Sir Syed University of Engineering and Technology, Karachi, Pakistan. He is working as a lecturer in the Department of Electronic Engineering, Sir Syed University of Engineering and Technology.

Wanyue Jiang is currently doing the $\mathrm{PhD}$ in control theory and control engineering from Nanjing University of Aeronautics \& Astronautics, Nanjing, China.

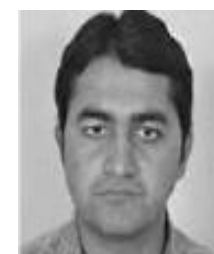

Muhammad Shafiq received his B.S. degree in electronic engineering from the Sir Syed University of Engineering and Technology, Karachi, Pakistan in 2011, and doing the M.S. in telecommunication engineering from Sir Syed University of Engineering and Technology, Karachi, Pakistan. He is working as a faculty member and researcher in the Department of Electronic Engineering, Sir Syed University of Engineering and Technology. 